\title{
Panhipopituitarismo y diabetes insípida central como primera manifestación de linfoma primario del sistema nervioso central en una paciente inmunocompetente
}

\author{
Edwin A. Wandurraga Sánchez $z^{1-3}$, William J. Morales Camacho ${ }^{2-3}$, Jessica E. Plata Ortiz².
}

\begin{abstract}
${ }^{1}$ Médico Internista Endocrinólogo. Universidad Autónoma de Bucaramanga - UNAB, Pontificia Universidad Javeriana - PUJ, Fundación Oftalmológica de Santander - Clínica Carlos Ardila Lulle, FOSCAL. Docente asociado, Universidad Autónoma de Bucaramanga - UNAB, Bucaramanga, Colombia.

${ }^{2}$ Médico Interno. Facultad de Ciencias de la Salud. Universidad Autónoma de Bucaramanga - UNAB, Bucaramanga, Colombia. ${ }^{3}$ Semillero de endocrinología y metabolismo. Facultad de ciencias de la salud. Universidad Autónoma de Bucaramanga - UNAB, Bucaramanga, Colombia.
\end{abstract}

Conflictos de intereses: Los autores declaran no tener conflicto de intereses.

Fecha de recepción: 14/09/2015

Fecha de aceptación: 4/11/2015

\section{Resumen}

$\mathrm{E}$ l panhipopituitarismo $(\mathrm{PH})$ hace referencia a la pérdida total de la función de la hipófisis anterior (adenohipófisis) que incluye los ejes somatótropo, tirótropo, corticótropo, gonadótropo, entre otros. La diabetes insípida central (DIC) no está contemplada en la definición de PH y su asociación simultánea implica la destrucción o degeneración de las neuronas localizadas en los núcleos supraóptico y paraventricular a nivel hipotalámico, es decir, un compromiso más extenso, dicha asociación se ha reportado hasta en $27 \%$ de los casos de linfoma primario del sistema nervioso central (LPSNC), la presencia de esta alteración obliga siempre a descartar enfermedades de carácter hematológico, infiltrativo, infeccioso y autoinmune. Presentamos el caso de una paciente de 19 años quien debutó con PH y DIC en la que no se pudo esclarecer un diagnóstico definitivo en su abordaje inicial, luego de casi tres años de evolución, una segunda biopsia realizada a nivel cerebral permitió confirmar el diagnóstico de linfoma primario del SNC. El curso lento y progresivo observado en nuestra paciente es propio de dicha neoplasia; sin embargo, el efecto citorreductor de los corticoides utilizados para el manejo de su déficit hormonal, favoreció el retraso en la identificación de esta enfermedad hematológica.

Palabras clave: panhipopituitarismo, diabetes insípida central, linfoma primario de sistema nervioso central, reporte de caso.

\section{Abstract}

The panhypopituitarism (PH) refers to total loss of function of the anterior pituitary (adenohypophysis), which ultimately can lead to commitment at differents endocrine axes involved at this level. Central diabetes insipidus (CDI) is not covered by the definition of $\mathrm{PH}$ and its simultaneous association involves the destruction or degeneration of neurons located in the supraoptic and paraventricular nuclei of the hypothalamus, it means a greater and more extent commitment. We present the case of a 19 years old female who debuted with PH and CDI in which failed to elucidate a definitive diagnosis in its initial approach. Finally, after nearly three years of evolution and a second biopsy performed in the brain the diagnosis of primary central nervous system lymphoma was confirmed.

Keywords: panhypopituitarism, Central diabetes insipidus, Primary Central Nervous System Lymphoma, Case report.

\section{Introducción}

El PH hace referencia a la pérdida total en la función de la adenohipófisis. La presencia simultánea de DIC sugiere un compromiso de mayor extensión a nivel central (hipotálamohipofisiario $)^{(1)}$. Distinguir entre una etiología de carácter neoplásico, infeccioso o infiltrativo constituye un importante reto diagnóstico en el ámbito clínico. A pesar de su baja incidencia el diagnóstico diferencial de LPSNC debe ser considerado en todo paciente que debuta con dichas manifestaciones endocrinas o en el que se documenta una afectación de uno o más ejes hormonales a nivel central. La supervivencia de los pacientes con LPSNC suele verse afectada debido a que en la mayoría de los casos el diagnóstico y tratamiento médico se realizan de forma tardía, debido principalmente a las características propias de dicha neoplasia(2).

\section{Reporte de caso}

Se trata de una paciente de 19 años previamente sana sin ningún antecedente de importancia, quien asistió a consulta externa por cuadro clínico de dos meses de evolución caracterizado por múltiples episodios eméticos, astenia, adinamia, 
polidipsia, poliuria, deterioro de la clase funcional de grandes a medianos esfuerzos, con posterior aparición de episodios de palpitaciones, cefalea holocraneana persistente, deposiciones diarreicas intermitentes, pérdida de peso (aproximadamente 6 kilogramos/2 meses) y amenorrea.

En los exámenes paraclínicos iniciales se evidenció un perfil hormonal compatible con panhipopituitarismo (PH) y diabetes insípida central (DIC); además se documentó hipostenuria, anemia y transaminitis (tabla 1). Se realizó resonancia nuclear magnética (RNM) de silla turca con resultado normal.

Al investigar la causa subyacente se realizaron estudios para descartar etiología infecciosa, hematológica y autoinmune con resultados negativos (excepto por ligera disminución del complemento) (tabla 1). La gammagrafía ósea corporal total y mielograma no tenían alteración. Se inició remplazo hormonal con prednisolona 7,5 mg/día, levotiroxina $125 \mathrm{mcg} /$ día y desmopresina $20 \mathrm{mcg}$ intranasal/día con buena evolución. La paciente egresó sin diagnóstico etiológico.

Tabla 1. Laboratorios realizados a la paciente

\begin{tabular}{|c|c|c|}
\hline Laboratorio & Resultado & Valor de Referencia \\
\hline TSH & 0,46 & $0,4-4,2 \mathrm{uUI} / \mathrm{ml}$ \\
\hline T4L & $0,75^{* *}$ & 0,93-1,7 ng/DI \\
\hline Cortisol 8am & $2,35 * *$ & $6,2-19,4 \mathrm{ug} / \mathrm{dl}$ \\
\hline FSH & $0,15^{* *}$ & $3,5-12,5 \mathrm{mUl} / \mathrm{ml}$ \\
\hline LH & $0,1 * *$ & 2,4-12,6 $\mathrm{mUl} / \mathrm{ml}$ \\
\hline Estradiol & $11,6 * *$ & $12,5-166 \mathrm{pg} / \mathrm{ml}$ \\
\hline Prolactina & $71 * *$ & $4,79-23,3 \mathrm{ng} / \mathrm{ml}$ \\
\hline Hemoglobina & $8,6 * *$ & $12,3-15,5 \mathrm{gr} / \mathrm{dl}$ \\
\hline VCM & 80,7 & $80-96 \mathrm{fl}$ \\
\hline HCM & 28,2 & $28-33 \mathrm{pg}$ \\
\hline Leucocitos & 6550 & $4400-11300 / \mathrm{mm}^{3}$ \\
\hline AST & $95 * *$ & $<32 \mathrm{U} / \mathrm{L}$ \\
\hline ALT & $154 * *$ & $<33 \mathrm{U} / \mathrm{L}$ \\
\hline Sodio & $149 * *$ & $135-148 \mathrm{mmol} / \mathrm{L}$ \\
\hline Potasio & 4,09 & $3,5-5,3 \mathrm{mmol} / \mathrm{L}$ \\
\hline Densidad urinaria & 1,005 & $1,010-1,025$ \\
\hline Creatinina & 0,88 & $0,50-0,90 \mathrm{mg} / \mathrm{dl}$ \\
\hline Glucemia & 78 & $70-99 \mathrm{mg} / \mathrm{dl}$ \\
\hline Lactato deshidrogenasa & 439 & $<480 \mathrm{UI} / \mathrm{L}$ \\
\hline Coombs directo & Negativo & Negativo \\
\hline Anticuerpos antinucleares & Negativo & Negativo \\
\hline C-ANCA & Negativo & Negativo \\
\hline P-ANCA & Negativo & Negativo \\
\hline Antimitocondriales & No reactivo & No reactivo \\
\hline Complemento - C3 & $85,7 * *$ & $90-180 \mathrm{mg} / \mathrm{dl}$ \\
\hline Complemento - C4 & 14,6 & $10-40 \mathrm{mg} / \mathrm{dl}$ \\
\hline ELISA HIV & No reactivo & No reactivo \\
\hline Hepatitis B, HBsAg & No reactivo & No reactivo \\
\hline Hepatitis B, Core IgM & No reactivo & No reactivo \\
\hline
\end{tabular}

**Resultado de laboratorio fuera de los valores de referencia del laboratorio.
Veinte meses después fue hospitalizada nuevamente por cuadro clínico de cefalea persistente y hemianopsia homónima izquierda, nuevo reporte de RMN cerebral evidenció lesión tumoral expansiva hipercaptante de $15 \times 18 \mathrm{~mm}$ localizada a nivel de núcleo lenticular y dorsal talámico derecho, además de silla turca vacía, sin desviación de línea media. Fue llevada a biopsia por neuroendoscopia guiada por neuronavegación, con posterior reporte de infiltrado linfocitario policlonal de predominio TCD8 negativo para linfoma. Dada la mejoría clínica con dexametasona a altas dosis, se decidió manejo ambulatorio multidisciplinario. En aquel momento se consideró sarcoidosis como diagnóstico diferencial.

Posteriormente en el seguimiento se decidió realización de PET/CT con 18-FDG, evidenciando nueva masa intraaxial intensamente hipermetabólica (SUV $=47,7)$ localizada en la sustancia blanca fronto-temporal izquierda que comprometía región núcleo-basal y que producía efecto de masa, edema vasogénico y ventriculomegalia contralateral (figura 1). Se realizó una segunda biopsia guiada por neuronavegación y abordaje transventricular, con reporte histopatológico de linfoma B difuso de célula grande (alto grado) primario del sistema nervioso central (LPSNC) CD20 (+) (figura 2), se dio quimioterapia con metotrexate, citarabina y carmustina (protocolo BAM), adicionalmente se realizó consolidación con trasplante autólogo de médula ósea. Presentó recaída tumoral al día 90 postrasplante, debido a esto se iniciaron cuatro ciclos de quimioterapia de rescate con metotrexate y citarabina en altas dosis $\left(3,5 \mathrm{~g} / \mathrm{m}^{2}, 2 \mathrm{~g} / \mathrm{m}^{2}\right.$ respectivamente), además se realizó radioterapia externa sobre sistema nervioso central con dosis total acumulada de 1600 centigray (cGy).

\section{Discusión}

Se analiza el caso de una mujer de 19 años quien debutó con PH y DIC sin encontrarse patología en el abordaje inicial. Casi tres años después se confirmó el diagnóstico de linfoma primario del SNC. La ausencia de tumor en imágenes cerebrales iniciales y el efecto citorreductor de los corticoides empleados en el tratamiento de la insuficiencia suprarrenal y el edema cerebral vasogénico, retrasó casi 3 años la realización de una segunda biopsia con el correspondiente diagnóstico definitivo. El curso lento y progresivo es usual en esta entidad.

El PH hace referencia a la pérdida total de la función de la hipófisis anterior (adenohipófisis). Desde el punto de vista bioquímico se puede identificar hipotiroidismo central (TSH normal o disminuida con T4L baja), insuficiencia adrenal secundaria (cortisol y ACTH bajo), hipogonadismo hipogonadotrópico (FSH, LH normal o bajas con estradiol bajo) y deficiencia de hormona de crecimiento. La DIC no se encuentra incluida en la definición de panhipopituitarismo y su presencia concomitante implica la destrucción o degeneración de las neuronas localizadas en los núcleos supraóptico y paraventricular a nivel hipota- 
Figura 1. PET/CT (tomografía con emisión de positrones con radiofármaco F-18 FDG.

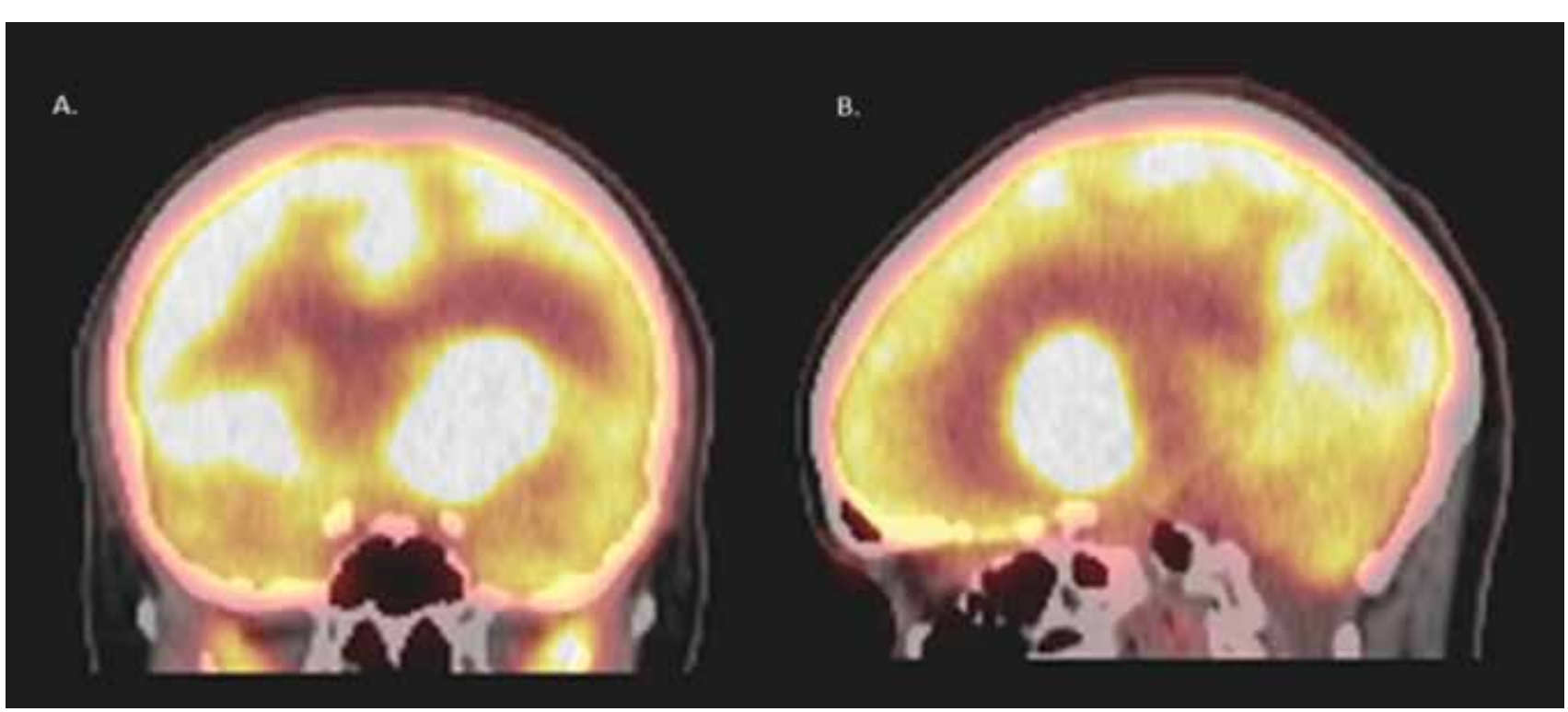

(A) Corte coronal. (B). Corte sagital. Se observa una masa intraaxial intensamente hipermetabólica (SUV: 47.7) localizada en la sustancia blanca fronto-temporal izquierda que compromete la región núcleo-basal y que produce efecto de masa, edema vasogénico y ventriculomegalia contralateral.

Figura 2. Reporte histopatológico de biopsia cerebral región núcleo basal izquierda

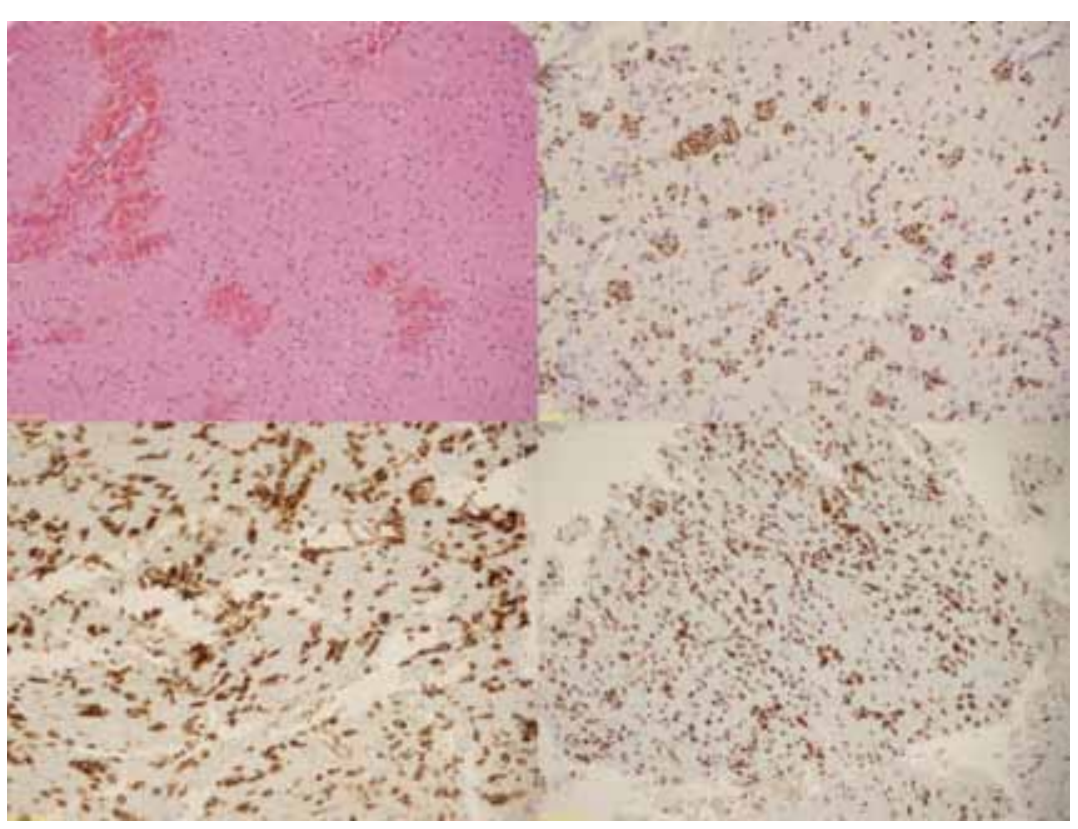

(A). Tumor linfoide constituido por células intermedias y grandes con numerosas mitosis y cuerpos apoptóticos. (B). Actividad proliferativa alta en zonas con mayor población tumoral (KI 67:100\%). (C). Inmunohistoquímica con células tumorales positivas para CD 20. (D). PAX 5, CD 10, BCL 6 y menos del $10 \%$ positivas para MUM 1 Y BCL 2; negativas para TDT y mieloperoxidasa. Diagnóstico: linfoma B difuso de célula grande (alto grado).

lámico ${ }^{(1)}$, es decir, un compromiso de mayor extensión. Las manifestaciones iniciales de estas alteraciones endocrinas son muy inespecíficas e inicialmente no suelen orientar hacia un diagnóstico claro, tal y como podemos evidenciar en nuestra paciente, durante el debut de su sintomatología dado por la presencia de náuseas, vómito, diarrea, cefalea, polidipsia, poliuria, que resultan ser manifestaciones secundarias al desequilibrio hormonal en los diferentes ejes endocrinológicos involucrados y que puede retrasar el diagnóstico definitivo del paciente que debuta con PH y DIC.

Existen pocos datos en la literatura respecto a las principales causas de PH y DIC. En un estudio realizado en población turca con 773 pacientes entre 16-84 años con deficiencia de una o más hormonas hipofisiarias se encontró que el 33,8\% tenían PH y el 12,6\% tenían DIC asociada. Dentro de las causas potenciales de PH y DIC se describieron craneofaringioma $(5,2 \%)$, histiocitosis $(0,3 \%)$, hipofisitis linfocítica en el 1,6\%, trauma craneoencefálico $(2,2 \%)$ y aspergiloma en el $0,3 \%{ }^{(3)}$. A nivel infeccioso, las entidades descritas con mayor frecuencia son la tuberculosis y la sífilis ${ }^{(4)}$. Los procedimientos quirúrgicos a nivel central junto con la radioterapia pueden ser etiologías adicionales que se deben tener en cuenta en el estudio de este par de alteraciones endocrinológicas $^{(4,5)}$.

El PH asociado a DIC se ha descrito en el $27 \%$ de los casos de LPSNC $^{(5)}$, los casos reportados en la literatura respecto a la presencia de estas manifestaciones clínicas como síntomas iniciales del LPSNC son escasos ${ }^{(6)}$. La presencia de un estado de inmunosupresión (congénito y/o adquirido), es el principal factor de riesgo relacionado con el desarrollo de esta neoplasia ${ }^{(14)}$, típicamente es una entidad en 
la que suele no evidenciarse la formación tumoral característica de muchas otras neoplasias, hecho que retrasa en innumerables ocasiones el diagnóstico y tratamiento oportuno de este tipo de linfoma ${ }^{(2)}$, afectando en últimas de manera significativa la supervivencia de los pacientes ${ }^{(7)}$. La edad de presentación oscila entre los 45 - 70 años, con una media de edad al momento del diagnóstico de 50 años aproximadamente ${ }^{(8)}$, aunque también se han reportado algunos casos en pacientes con menor edad (promedio de 14 años) ${ }^{(9)}$. En nuestro caso en particular, se trata de una paciente que no cursaba con ningún grado de inmunodeficiencia, por lo que la presencia de dicha condición no es un aspecto necesario en la totalidad de los casos para el desarrollo del LPSNC, adicionalmente llama la atención la corta edad de nuestra paciente, comparada con el rango de edad que se ha reportado con mayor frecuencia en la literatura.

Con respecto a los estudios adicionales realizados en nuestra paciente para determinar la etiología definitiva que explicase el compromiso endocrinológico a nivel hipotalámico e hipofisiario, la tomografía con emisión de positrones con radiofármaco F-18 FDG (PET/CT) fue la herramienta que permitió en última instancia la identificación y localización de la lesión blanco para realizar la segunda biopsia que confirmó el diagnóstico de LPSNC. La importancia de dicho estudio radica en la capacidad que posee para identificar un compromiso sistémico en pacientes con enfermedad neoplásica hematológica confirmada ${ }^{(10)}$. En nuestro caso se puede observar una evolución desafortunada y poco favorable que podría estar en relación con la alta actividad proliferativa tumoral expresada en la tomografía con F-18 FDG.

Pese a que el LPSNC presenta sensibilidad a la quimio y radioterapia ${ }^{(13)}$, el pronóstico sigue siendo poco favorable con tiempos de supervivencia que oscilan entre 15 y 45 meses en aquellos pacientes que reciben tratamiento ${ }^{(11)}$, esto puede deberse tanto a la agresividad que per se posee esta neoplasia ${ }^{(7,12)}$, como al diagnóstico tardío ${ }^{(2)}$. La evolución de nuestra paciente fue poco favorable dada la presencia de recaída tumoral temprana posterior a dos ciclos de quimioterapia y trasplante autólogo de médula ósea, que determinó el inicio de terapia de rescate con metotrexate y citarabina en altas dosis, así como la utilización de radioterapia externa holoencefálica.

\section{Referencias}

1. Mohamad M, Cosi G,Genovese E, Manca-Bitti ML,Cohen A, Et all.Central diabetes insipidus in children and young adults.N Engl J Med. $2000 ;(343): 998-1007$.

2. Akhtar S, Cheesman E,Jude EB. SIADH and partial hypopituitarism in a patient with intravascular large B-cell lymphoma: a rare cause of a common presentation. BMJ Case Rep. 2013 Jan 28; 2013.

3. Tanriverdi F, Dokmetas HS,Kebapcı N,Kilicli F, Atmaca H, Et all.Etiology of hypopituitarism in tertiary care institutions in Turkish population: analysis of 773 patients from pituitary study group database.Endocrine.2014;(47):198-205.

4. Toogood AA,Stewart PM.Hypopituitarism: Clinical Features, Diagnosis, and Management. Endocrinol Metab Clin N Am. 2008; (37): 235-261.

5. Giustina A, Gola M, Doga M, Rosei EA. Clinical review 136: Primary lymphoma of the pituitary: an emerging clinical entity. J Clin Endocrinol Metab.2001; (86):4567-4575.

6. Layden BT, Dubner S, Toft DJ, Kopp P, Grimm S, Et al.Primary CNS Lymphoma with Bilateral Symmetric Hypothalamic Lesions Presenting with Panhypopituitarism and Diabetes Insipidus. Pituitary. 2011; 14(2): 194-197.

7. Kasenda B, Haug V, Schorb E, Fritsch K, Finke J, Et al. 18F-FDG PET Is an Inde-
En definitiva el trasfondo del abordaje del paciente con PH y DIC lo constituye no sólo la suplencia hormonal, sino los estudios adicionales (laboratorios y/o imágenes diagnósticas) que permitan determinar la etiología que explique el compromiso endocrinológico a nivel central para iniciar el tratamiento respectivo lo antes posible.

\section{Conclusiones}

La asociación entre PH y DIC denota un compromiso importante a nivel hipotalámico e hipofisiario que pudiera ser explicado por condiciones autoinmunes, infecciosas, infiltrativas y/o neoplásicas. El abordaje de estas alteraciones endocrinas no sólo implica la suplencia hormonal necesaria para mejorar la sintomatología clínica del paciente, sino que amerita un estudio exhaustivo con el fin de realizar un diagnóstico preciso y definitivo. El diagnóstico etiológico muchas veces se ve retrasado por las manifestaciones poco floridas e inespecíficas en el paciente con dichas alteraciones. Sumado a esto, el efecto citorreductor e inmunosupresor de la terapia con glucocorticoides empleada durante el tratamiento, puede retrasar aún más el diagnóstico debido a la mejoría clínica posterior al inicio de dicha medicación. Las neoplasias hematológicas y más específicamente el LPSNC deben estar siempre como diagnóstico diferencial en todo paciente que debuta con PH y DIC o en quien se documenta un compromiso hormonal de origen central, incluso en ausencia de inmunosupresión. Dicha asociación se ha reportado en $27 \%$ de los casos de LPSNC. El pronóstico del LPSNC continúa siendo pobre con altas cifras de recaída tumoral y bajos índices de supervivencia a corto y mediano plazo. Finalmente, se puede considerar que el PH asociado a DIC son manifestaciones apenas incipientes de condiciones más complejas, en la mayoría de los casos, que deben ser investigadas y diagnosticadas en el menor tiempo posible.

\section{Agradecimientos}

Agradecemos a la Dra. Isabel Bolívar por su ayuda en la obtención de las imágenes de patología.

pendent Outcome Predictor in Primary Central Nervous System Lymphoma. THE JOURNAL OF NUCLEAR MEDICINE. 2013; 54(2):184-91.

8. Braaten KM, Betensky RA, de Leval L, et al. BCL6 expression predicts improved survival in patients with primary central nervous system lymphoma. Clin Cancer Res. 2003;(9):1063-1069.

9. Abla O, Weitzman S, Blay JY, et al. Primary CNS lymphoma in children and adolescents: a descriptive analysis from the International Primary CNS Lymphoma Collaborative Group (IPCG). Clin Cancer Res. 2011;(17):346-352.

10. Mohile NA, DeAngelis LM, Abrey LE. The utility of body FDG PET in staging primary central nervous system lymphoma. Neuro Oncol. 2008;10(2):223-228.

11. Coulter I, Garrioch S ,Toft A. An atypical cause of trigeminal neuralgia and Panhypopituitarism.The British Journal of Radiology.2010; (83): 1087-1089.

12. Singh AD, Lewis $\mathrm{H}$, Schachat AP. Primary lymphoma of the central nervous system. Ophthalmol Clin North Am. 2005; (18): 199-207.

13. Mohile NA, Deangelis LM, Abrey LE. Utility of brain FDG-PET in primary CNS lymphoma. Clin Adv Hematol Oncol. 2008;6(11):818-20, 840.

14. Cote TR, Manns A, Hardy CR, Yellin FJ, Hartge P. Epidemiology of brain lymphoma among people with or without acquired immunodeficiency syndrome. AIDS/Cancer Study Group. J Natl Cancer Inst 1996; (88): 675-679. 